Kragujevac Journal of Mathematics

Volume 44(3) (2020), Pages 415-421.

\title{
NUMERICAL RADIUS INEQUALITIES IN 2-INNER PRODUCT SPACES
}

\author{
PANACKAL HARIKRISHNAN ${ }^{1}$, HAMID REZA MORADI ${ }^{2}$, \\ AND MOHSEN ERFANIAN OMIDVAR ${ }^{3}$
}

\begin{abstract}
In this paper, we have obtained the analogue results on numerical radius inequalities from the classical inner product spaces to 2-inner product spaces. We have established several related reverse inequalities and some well known results in 2-inner product spaces.
\end{abstract}

\section{Introduction AND PRELIMINARIES}

Let $\mathscr{X}$ be a linear space of dimension greater than 1 over the field $K=\mathbb{R}$ of real numbers or the field $K=\mathbb{C}$ of complex numbers. Suppose that $(\cdot, \cdot \cdot \cdot)$ is a $K$-valued function defined on $\mathscr{X} \times \mathscr{X} \times \mathscr{X}$ satisfying the following conditions:

(I1) $(x, x \mid z) \geq 0$, and $(x, x \mid z)=0$ if and only if $x$ and $z$ are linearly dependent;

(I2) $(x, x \mid z)=(z, z \mid x)$;

(I3) $(y, x \mid z)=\overline{(x, y \mid z)}$;

(I4) $(\alpha x, y \mid z)=\alpha(x, y \mid z)$ for any scalar $\alpha \in K$;

(I5) $\left(x+x^{\prime}, y \mid z\right)=(x, y \mid z)+\left(x^{\prime}, y \mid z\right)$.

$(\cdot, \cdot \mid \cdot)$ is called a 2 -inner product on $\mathscr{X}$ and $(\mathscr{X},(\cdot, \cdot \mid \cdot))$ is called a 2 -inner product space (or 2-pre-Hilbert sapce). Some basic properties of 2 -inner product $(\cdot, \cdot \cdot \cdot)$ can be immediately obtained as follows (see [3]):

$(\mathrm{P} 1)(0, y \mid z)=(x, 0 \mid z)=(x, y \mid 0)=0$

(P2) $(x, \alpha y \mid z)=\bar{\alpha}(x, y \mid z)$;

(P3) $(x, y \mid \alpha z)=|\alpha|^{2}(x, y \mid z)$, for all $x, y, z \in \mathscr{X}$ and $\alpha \in K$.

Key words and phrases. 2-inner product space, linear 2-normed space, numerical range, numerical radius.

2010 Mathematics Subject Classification. Primary: 46C50. Secondary: 47A12, 47L30, 47A30.

DOI 10.46793/KgJMat2003.415H

Received: April 21, 2017.

Accepted: May 26, 2018. 
Using the above properties, one has proved that Cauchy-Schwartz inequality (see [5])

$$
|(x, y \mid z)|^{2} \leq(x, x \mid z)(y, y \mid z) .
$$

It should be noticed that, the most standard example for a linear 2-inner product $(\cdot, \cdot \mid \cdot)$ is defined on $\mathscr{X}$ by

$$
(x, y \mid z):=\operatorname{det}\left(\begin{array}{cc}
\langle x, y\rangle & \langle x, z\rangle \\
\langle z, y\rangle & \langle z, z\rangle
\end{array}\right),
$$

for all $x, y, z \in \mathscr{X}$. In [2], it is shown that, in any given 2 -inner product space $(\mathscr{X},(\cdot, \cdot \mid \cdot))$, we can define a function

$$
\|x, z\|=\sqrt{(x, x \mid z)}
$$

for all $x, z \in \mathscr{X}$. It is not hard to see that this function satisfies the following conditions (see [6]):

(N1) $\|x, y\|=0$ if and only if $x$ and $y$ are linearly dependent;

(N2) $\|x, y\|=\|y, x\|$;

(N3) $\|\alpha x, y\|=|\alpha|\|x, y\|$ for any real number $\alpha$;

(N4) $\|x, y+z\| \leq\|x, y\|+\|x, z\|$.

Any function $\|\cdot, \cdot\|$ defined on $\mathscr{X} \times \mathscr{X}$ and satisfying the above conditions is called a 2 -norm induced from a 2 -inner product on $\mathscr{X}$ and $(\mathscr{X},\|\cdot, \cdot\|)$ is called linear 2-normed space.

Some of the basic properties of 2-norms are that they are non-negative and $\|x, y+\alpha x\|=\|x, y\|$, for all $x, y \in \mathscr{X}$ and all $\alpha \in \mathbb{R}$. Whenever a 2-inner product space $(\mathscr{X},(\cdot, \cdot \mid \cdot))$ is given, we consider it as a linear 2 -normed space $(\mathscr{X},\|\cdot, \cdot\|)$ with the 2-norm defined by (1.2).

An operator $A \in \mathcal{B}(\mathscr{X})$ is said to be bounded if there exists a real number $M>0$ such that

$$
\|A x, y\| \leq M\|x, y\|,
$$

for every $x, y \in \mathscr{X}$. The norm of the $b$-operator is defined by [9]:

$$
\|A\|_{b}=\sup \{\|A x, b\|:\|x, b\|=1\},
$$

where $b$ is fixed element in $\mathscr{X}$. We can easily verify that the left-hand side of (1.3), is equivalent with $\sup \{|(A x, x \mid b)|:\|x, b\| \leq 1\}$.

Harikrishnan et al. in [8] proved the Riesz theorem in 2-inner product spaces. As a consequence of their work, we have

$$
(A x, y \mid b)=\left(x, A^{*} y \mid b\right),
$$

for each $x, y \in \mathscr{X}$ and fixed element $b \in \mathscr{X}$.

Recently, M. E. Omidvar et al. [10] established various reverses of the CauchySchwarz and triangle inequalities in 2-inner product spaces. 
In this paper, we introduce the concepts of $b$-numerical radius in 2 -inner product spaces. Some fundamental inequalities related to the $b$-numerical radius of bounded linear operators in 2-inner product spaces are established.

\section{Main Results}

We first review some basic facts about numerical range and numerical radius in Hilbert space $\mathscr{H}$, then try to define them in a 2 -inner product space. Let $(\mathscr{H},\langle\cdot, \cdot\rangle)$ be a complex Hilbert space and $\mathcal{B}(\mathscr{H})$ denote the $C^{*}$-algebra of all bounded linear operators on $\mathscr{H}$. An operator $A \in \mathcal{B}(\mathscr{H})$ is called positive if $\langle A x, x\rangle \geq 0$ for all $x \in \mathscr{H}$. We write $A \geq 0$ if $A$ is positive. The numerical radius is defined by

$$
\omega(A)=\sup \{|\lambda|: \lambda \in W(A)\},
$$

where $W(A)$ is the numerical range of $A$ given by

$$
W(A)=\{\langle A x, x\rangle: x \in \mathscr{H},\|x\|=1\} .
$$

The following properties of $W(A)$ are immediate:

(a) $W(\alpha I+\beta A)=\alpha+\beta W(A)$ for $\alpha, \beta \in \mathbb{C}$;

(b) $W\left(A^{*}\right)=\{\bar{\lambda}: \lambda \in W(A)\}$, where $A^{*}$ is the adjoint operator of $A$;

(c) $W\left(U^{*} A U\right)=W(A)$ for any unitary operator $U$.

The most important classical fact about the geometry of the numerical range is that it is convex and its closure contains the spectrum of the operator. The usual operator norm of $A$, is defined by

$$
\|A\|=\sup _{\|x\|=1}\|A x\|, \quad \text { for all } x \in \mathscr{H},
$$

where $\|x\|=\langle x, x\rangle^{\frac{1}{2}}$. It is well known that $\omega(\cdot)$ defines a norm on $\mathcal{B}(\mathscr{H})$ and that for every $A \in \mathcal{B}(\mathscr{H})$, we have

$$
\frac{1}{2}\|A\| \leq \omega(A) \leq\|A\| .
$$

Thus, the usual operator norm and the numerical radius norm are equivalent. See [7] for a discussion and further references.

Now we are in a position to state the main result of this section. The $b$-numerical range of $A \in \mathcal{B}(\mathscr{X})$, denoted by $W_{b}(A)$, is the subset of the complex numbers given by

$$
W_{b}(A)=\{(A x, x \mid b):\|x, b\| \leq 1\} .
$$

The $b$-numerical radius of $A \in \mathcal{B}(\mathscr{X})$, denoted by $\omega_{b}(A)$, is defined by

$$
\omega_{b}(A)=\sup \{|(A x, x \mid b)|:\|x, b\| \leq 1\} .
$$

It is easy to see that, for any $(x, b) \in \mathscr{X} \times\langle b\rangle$, we have

$$
|(A x, x \mid b)| \leq \omega_{b}(A)\|x, b\|^{2} .
$$


The $b$-numerical radius $\omega_{b}(A)$ of an operator $A$ on $\mathscr{X}$ is a norm on $\mathcal{B}(\mathscr{X})$, this norm is equivalent to the $b$-operator norm. In order to get our main result, we need the following lemmas:

Lemma 2.1 ([1]). Let $A \in \mathcal{B}(\mathscr{X})$, then

$$
\begin{aligned}
4(A x, y \mid z)= & (A(x+y), x+y \mid z)-(A(x-y), x-y \mid z) \\
& +i(A(x+i y), x+i y \mid z)-i(A(x-i y), x-i y \mid z),
\end{aligned}
$$

for any $x, y, z \in \mathscr{X}$.

Lemma $2.2([4])$. For every $x, y \in \mathscr{X}$, we have

$$
\|x+y, b\|^{2}+\|x-y, b\|^{2}=2\left(\|x, b\|^{2}+\|y, b\|^{2}\right) .
$$

We shall, however, present another result, which is a possible generalization of (2.1).

Proposition 2.1. For each $A \in \mathcal{B}(\mathscr{X})$, we get

$$
\frac{1}{2}\|A\|_{b} \leq \omega_{b}(A) \leq\|A\|_{b}
$$

Proof. If $\lambda=(A x, x \mid b)$ with $\|x, b\| \leq 1$, by Schwartz inequality we obtain

$$
|\lambda| \leq|(A x, x \mid b)| \leq\|A x, b\|\|x, b\| \leq\|A\|_{b} .
$$

On the other hand, by Lemma 2.1 and Lemma 2.2 we get

$$
\begin{aligned}
4|(A x, y \mid b)| & \leq \omega_{b}(A)\left[\|x+y, b\|^{2}+\|x-y, b\|^{2}+\|x+i y, b\|^{2}+\|x-i y, b\|^{2}\right] \\
& =2 \omega_{b}(A)\left[\|x, b\|^{2}+\|y, b\|^{2}+\|x, b\|^{2}+\|i y, b\|^{2}\right] \\
& \leq 8 \omega_{b}(A) .
\end{aligned}
$$

By taking supremum over $\|x, b\|=\|y, b\|=1$, we deduce the desired result.

Theorem 2.1. Let $A, B \in \mathcal{B}(\mathscr{X})$ and $A B=B A$, then

$$
\omega_{b}(A B) \leq 2 \omega_{b}(A) \omega_{b}(B) .
$$

Proof. We may assume $\omega_{b}(A)=\omega_{b}(B)=1$ and show that $\omega_{b}(A B) \leq 2$. By the triangle inequality, the power inequality theorem, and the subadditivity of $\omega(\cdot)$, we have

$$
\begin{aligned}
\omega_{b}(A B) & \equiv \omega_{b}\left(\frac{1}{4}\left[(A+B)^{2}-(A-B)^{2}\right]\right) \\
& \leq \frac{1}{4} \omega_{b}\left[(A+B)^{2}-(A-B)^{2}\right] \\
& \leq \frac{1}{4}\left[\left(\omega_{b}(A+B)\right)^{2}+\left(\omega_{2}(A-B)\right)^{2}\right] \\
& \leq \frac{1}{4}\left[\left(\omega_{b}(A)+\omega_{b}(B)\right)^{2}+\left(\omega_{b}(A)+\omega_{b}(B)\right)^{2}\right] \\
& =2
\end{aligned}
$$

as desired. 
The following simple result provides a connection between the numerical radius and $b$-numerical radius as follows:

Theorem 2.2. Let $A \in \mathcal{B}(\mathscr{X})$, then

$$
\omega(A) \leq \omega_{b}(A)+\|A\|_{b}^{\prime},
$$

where

$$
\|A\|_{b}^{\prime}=\sup \{|(A x, x \mid b)|:\|x, b\| \leq 1\}
$$

and $b \in \mathscr{X}$ is a fixed element.

Proof. We observe that

$$
\begin{aligned}
|(A x, x \mid b)| & =\left|(A x, x)\|b\|^{2}-(A x, b)(b, x)\right| \quad(\text { by }(1.1)) \\
& \geq|(A x, x)|\|b\|^{2}-|(A x, b)||(b, x)| .
\end{aligned}
$$

By taking supremum over $\|x, b\| \leq 1$ we deduce the desired result (2.2).

The following inequalities may be stated as well.

Theorem 2.3. Let $A \in \mathcal{B}(\mathscr{X})$ be a bounded linear operator on the linear 2-normed space $\mathscr{X}$. If $\lambda \in \mathbb{C} \backslash\{0\}$ and $\alpha>0$ are such that

$$
\|A-\lambda I\|_{b} \leq \alpha
$$

where $I$ is the identity operator on $\mathscr{X}$, then

$$
\|A\|_{b}-\omega_{b}(A) \leq \frac{1}{2} \frac{\alpha^{2}}{|\lambda|}
$$

Proof. For $(x, b) \in \mathscr{X},\langle b\rangle$ with $\|x, b\|=1$, we have from (2.3) that

$$
\|(A-\lambda) x, b\| \leq\|A-\lambda I\|_{b} \leq \alpha,
$$

giving

$$
\begin{aligned}
\|A x, b\|^{2}+|\lambda|^{2} & \leq 2 \operatorname{Re}[\bar{\lambda}(A x, x \mid b)]+\alpha^{2} \\
& \leq 2|\lambda|(A x, x \mid b)+\alpha^{2} .
\end{aligned}
$$

Taking supremum over $(x, b) \in \mathscr{X},\langle b\rangle$, with $\|x, b\|=1$ we get the following inequality

$$
\|A\|_{b}^{2}+|\lambda|^{2} \leq 2 \omega_{b}(A)|\lambda|+\alpha^{2} .
$$

Since

$$
2\|A\|_{b}|\lambda| \leq\|A\|_{b}^{2}+|\lambda|^{2}
$$

hence by (2.6) and (2.7) we deduce the desired inequality (2.4).

Corollary 2.1. In particular, if $\|A-\lambda I\|_{b} \leq \alpha$ and $|\lambda|=\omega_{b}(A), \lambda \in \mathbb{C}$, then

$$
\|A\|_{b}-\omega_{b}^{2}(A) \leq \alpha^{2} \text {. }
$$


Proposition 2.2. Let $A \in \mathcal{B}(\mathscr{X})$ be a non zero bounded linear operator on the linear 2-normed space $\mathscr{X}$ and $\lambda \in \mathbb{C} \backslash\{0\}$ and $\alpha>0$ with $|\lambda|>\alpha$. If

$$
\|A-\lambda I\|_{b} \leq \alpha
$$

then

$$
\sqrt{1-\frac{\alpha^{2}}{|\lambda|^{2}}} \leq \frac{\omega_{b}(A)}{\|A\|_{b}}
$$

Proof. From (2.6) of Theorem 2.3, we have

$$
\|A\|_{b}^{2}+|\lambda|^{2}-\alpha^{2} \leq 2|\lambda| \omega_{b}(A),
$$

which implies, on dividing with $\sqrt{|\lambda|^{2}-\alpha^{2}}>0$ that

$$
\frac{\|A\|_{b}^{2}}{\sqrt{|\lambda|^{2}-\alpha^{2}}}+\sqrt{|\lambda|^{2}-\alpha^{2}} \leq \frac{2|\lambda| \omega_{b}(A)}{\sqrt{|\lambda|^{2}-\alpha^{2}}} .
$$

Whence

and by (2.9) we deduce

$$
2\|A\|_{b} \leq \frac{\|A\|_{b}^{2}}{\sqrt{|\lambda|^{2}-\alpha^{2}}}+\sqrt{|\lambda|^{2}-\alpha^{2}}
$$

which is equivalent to $(2.8)$.

$$
\|A\|_{b} \leq \frac{\omega_{b}(A)|\lambda|}{\sqrt{|\lambda|^{2}-\alpha^{2}}}
$$

Corollary 2.2. Squaring (2.8), we get the inequality

$$
\|A\|_{b}^{2}-\omega_{b}^{2}(A) \leq \frac{\alpha^{2}}{|\lambda|^{2}}\|A\|_{b}^{2} .
$$

Corollary 2.3. Let $A \in \mathcal{B}(\mathscr{X})$ be a bounded linear operator on the linear 2-normed space and $\lambda \in \mathbb{C} \backslash\{0\}$ and $\alpha>0$ with $|\lambda|>\alpha$ then $-\frac{\sqrt{3}}{2} \leq \frac{\alpha}{|\lambda|} \leq \frac{\sqrt{3}}{2}$.

Proof. From Proposition 2.1, we infer that $\frac{1}{2} \leq \frac{\omega_{b}(A)}{\|A\|_{b}}$.

By (2.8) we have $\sqrt{1-\frac{\alpha^{2}}{|\lambda|^{2}}} \leq \frac{\omega_{b}(A)}{\|A\|_{b}}$. Combining the above two inequalities one can obtain $\sqrt{1-\frac{\alpha^{2}}{|\lambda|^{2}}} \geq \frac{1}{2}$ implies $\left(\frac{\alpha}{|\lambda|}\right)^{2} \leq \frac{3}{4}$, which implies $-\frac{\sqrt{3}}{2} \leq \frac{\alpha}{|\lambda|} \leq \frac{\sqrt{3}}{2}$.

Acknowledgements. The authors are grateful to the anonymous referees for their valuable suggestions. The first author acknowledges MIT, Manipal Academy of Higher Education (Deemed to be University), India and the other authors acknowledges Department of Mathematics, Islamic Azad University, Mashhad, Iran for their kind encouragement. 


\section{REFERENCES}

[1] Y. Cho, C. Lin, S. Kim and A. Misiak, Theory of 2-inner Product Spaces, Nova Science Pub Inc, Huntington, 2001.

[2] C. Diminnie, 2-inner product spaces, Demonstr. Math. 6 (1973), 525-536.

[3] S. S. Dragomir, Y. J. Cho, S. S. Kim and A. Sofo, Some Boas-Bellman type inequalities in 2-inner product spaces, Journal of Inequalities in Pure and Applied Mathematics 6(2) (2005), Article ID 55.

[4] R. Ehret, Linear 2-Normed Spaces, Doctoral Dissertation, Saint Louis University, 1968.

[5] R. Freese, S. S. Dragomir, Y. J. Cho and S. S. Kim, Some companions of gruss inequality in 2-inner product spaces and applications for determinantal integral inequalities, Commun. Korean Math. Soc. 20 (2005), 487-503.

[6] R. W. Freese and Y. J. Cho, Geometry of Linear 2-Normed Spaces, Nova Science Publishers, New York, 2001.

[7] K. E. Gustafson and D. K. Rao, Numerical range, in: Numerical Range, Springer-Verlag, New York, 1997, 1-26.

[8] P. Harikrishnan, P. Riyas and K. Ravindran, Riesz theorems in 2-inner product spaces, Novi Sad J. Math. 41 (2011), 57-61.

[9] Z. Lewandowska, Linear operators on generalized 2-normed spaces, Bull. Math. Soc. Sci. Math. Roumanie (N.S.) 42 (1999), 353-368.

[10] M. E. Omidvar, H. R. Moradi, S. S. Dragomir and Y. J. Cho, Some reverses of the CauchySchwarz and triangle inequalities in 2-inner product spaces, Kragujevac J. Math. 41 (2017), $81-92$.

${ }^{1}$ Department of Mathematics,

Manipal Institute of Technology,

Manipal Academy of Higher Education,

MANIPAL, INDIA

Email address: pkharikrishnans@gmail.com, pk.harikrishnan@manipal.edu

${ }^{2}$ Young Researchers and Elite Club,

MashHad Branch, Islamic Azad University,

MASHHAD, IRAN

Email address: hrmoradi@mshdiau.ac.ir

${ }^{3}$ Department of Mathematics,

ISLAMIC AZAD UNIVERSITY,

MASHHAD, IRAN

Email address: erfanian@mshdiau.ac.ir 